\title{
Morphofunctional aspects of clefts and their repercussions on growth
}

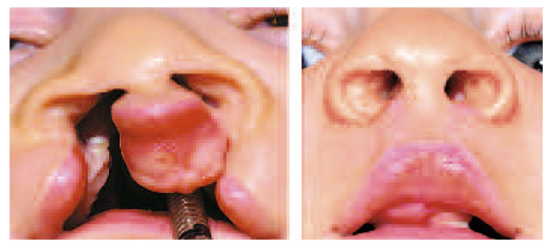

Rehabilitation of nasal ventilation accomplished in the initial operations

\author{
Jean-Claude TALMANT, Jean-Christian \\ TALMANT, Jean-Pierre LUMINEAU
}

\begin{abstract}
Over the last 50 years, the dominant conception of the etiology of facial clefts postulated that they resulted from a mesodermal deficit that accounted for the primary hypoplasia and the unpredictable nature of subsequent oro-facial growth. Because of this notion, rigid dogmatic interdictions, with no allowance for nuanced treatment adjustments were issued banning sub-periosteal and sub-perichondrial dissections as well as the undertaking of any nasal surgery during the growth period. These prohibitions have had a grave braking effect on the development of treatment for cleft lip and palate patients.

With a new comprehension of the important role that the dynamics of fetal ventilation plays in the development of the airways and its influence on maxillary growth researchers no longer have any doubt that normal growth mechanisms operating under abnormal anatomic conditions created by the cleft are the actual causes of the malformations and the facial growth deficit suffered by children born with clefts. It now seems likely that the nasal septum plays only a passive role in growth, as Moss believed, rather than the active one that Scott as well as Delaire defined, calling it the driving force of maxillary development. They advocated re-inserting the lateral muscles on the anterior nasal spine. But practitioners who limit their functional approach to this procedure risk being deceived. In addition this focusing on muscles distracts us from what is essential, nasal ventilation, whose rehabilitation beginning with the first operation and preservation throughout treatment are absolutely necessary to ensure the normal unfolding of a cleft palate patient's facial growth.
\end{abstract}

Address for correspondence:

\section{J.C. TALMANT}

Centre de compétence de traitement, Des fentes labio-maxill0-palatine des Pays-de-Loire,

Clinique Jules Verne. 2 route de Paris, 44300 Nantes.

jeanclaude.talmant@libertysurf.fr 
To achieve this goal surgeons must complete anatomic reconstruction with precisely timed, ambitious surgery carefully designed to control scar formation. Whether their procedure is unilateral or bilateral surgeons make this correction of the nasal malformation best if they operate when patients are six months old and close the lip defect at the same time. During this procedure surgeons close the soft palate defect using the Sommerlad technique taking advantage of the shrinking of the hard palate gap that closes enough to allow for simple, complete surgical elimination of the cleft in two layers without leaving dense scar tissue or denuded areas. By the time patients are 4 years old orthodontists have expanded the anterior maxilla to establish good canine function and surgeons can close the alveolar cleft with a gingivoperiosteoplasty and an iliac graft. Each procedure conserves the width of the piriform orifice, the nasal fossae and the anterior, incisor-canine sector of the arch. Nasal ventilation, as well as symmetrical, centered mastication, is required for normal facial growth.

\section{KEYWORDS}

\author{
Cleft lip, \\ Cleft palate, \\ Maxillo-facial development, \\ Maxilla,
}

Nose.
Received: 05-2011. Accepted: 08-2011.

\section{1 - INTRODUCTION}

So many patients with labio-maxillopalatal clefts have facial growth problems that treatment teams adopt a kind of fatalism that provokes them to make damaging therapeutic compromises of a surprising diversity.

From the time surgeons perform their first operation on infant cleft lip and palate patients they make a commitment to the future of these children. They must supervise the indispensable smooth collaboration of a multi-disciplinary team that is enlisted for the long term. Each member must share the conception of early treatment to rehabilitate different orofacial functions, especially nasal ventilation and mastication, and, in this framework, strive to restore maxillary, oral, and nasal structures by reshaping 
them into normal morphology of adequate proportions.

In this context, it is worth remembering anatomical fundamentals that are often overlooked: the width of the palatal vault is identical to that of the nasal floor and the width of the incisorcanine sector equals the width of the piriform orifice and of the nasal valve!

\section{2 - THE FUNCTIONAL BASIS OF TREATMENT OF CLEFTS}

If the treatment of clefts were simple we would have long known how to proceed most effectively, but generations of surgeons have struggled to find their way in this difficult field.

Those who have selected restoration of function as their primary goal have without doubt made the most useful advancements: "We can find all the normal elements on the borders of clefts, modified only by the presence of the cleft itself... Their repair should rightfully be the province of embryological surgery...We are obliged to identify these altered structures so that we can place them in correct positions without substituting other tissues that would not give the same result..." We can easily imagine that Victor Veau ${ }^{29}$ was talking about functional anatomy in 1928 in those few sentences.

Jean Delaire ${ }^{4}$, who, together with Scott ${ }^{16}$, thought that the nasal septum is the driving force of anterior and vertical facial growth has emphasized the importance of restoring the continuity of the labio-nasal muscles that the cleft had ruptured and of their balanced placement on the septum and the anterior nasal spine.

Nevertheless, 36 years of experience have shown us that by concentrating all our attention on muscle repair, this procedure lends an exces- sive importance to the "septal traction" concept of facial growth, and that despite its supposed functional effects it does not significantly reduce the frequency of the occurrence of impaired maxillary growth ${ }^{15}$. From this data, a number of authors conclude that these abnormalities are an inherent component of the malformation whose growth potential is "limited and unpredictable"13.

Readers of the works of OrtizMonasterio $^{12}$, published 50 years, cannot help being troubled by them. He examined a large number of adults who had received no surgical correction of their clefts and found that their maxillary growth was comparable to that of the normal, general population. This disconcerting finding was widely and stingingly rejected. Still, the malformations of adults who had not been operated on are accompanied by poor oro-nasal functioning and yet their facial growth is better than that of patients who had surgery!

In 2007, utilizing the most up-to-date methods, Enrina Diah ${ }^{7}$ discerned in adults who had not been operated on an intrinsic deficiency on both sides of the cleft anterior to the premolars, but with sagittal development comparable to what is found in the general population and no loss of maxillary inter-molar width. All of this makes clear what Victor Veau predicted and what Jean 
Delaire affirmed that cleft palate patients retain normal growth potential that is constrained to operate under abnormal conditions.

Of all functions nasal ventilation is probably the one most disturbed in cleft palate patients, but it is also the one most widely neglected by surgeons, even those deeply concerned with restoring function, who focus their efforts on muscle repair. The prohibitions against performing subperiosteal and sub-perichondrial dissections as well as early nasal surgery in children still in the growth period have so stifled the curiosity of treatment teams that the issue of fetal, post-natal, and infantile nasal breathing has remained an unexplored domain. It seems to us that it is time to take an interest in it.

\section{3 - THE DEVELOPMENT OF LABIO-MAXILLARY-PALATAL CLEFTS IN UTERO}

The work of Jacques Talmant has greatly clarified our understanding of the malformations observed in newborns with unilateral or bilateral clefts as logical consequences of modifications of the facial envelope and of the dynamics of fetal ventilation caused by the cleft.

\section{3 - 1 - The mechanics of the facial envelope ${ }^{19}$}

The normal arrangement of the muscles of the face closely follows a diagrammatic representation of the tensions within the facial envelope. A rupture of the envelope that follows development of a cleft lip changes the distribution of tensions and alters the distribution of muscles. The nasalis (compressor naris) muscle ${ }^{24}$, which has two heads, the transverse and the myrtiform, is the most severely affected. The only muscle of the Superficial Muscular Aponeurotic System that has two fixed insertions, the nasilis becomes, after its rupture, the envelope's anterior an- chorage point on the borders of the cleft. Because of this attachment, the nasalis seems to resist the backward movement of other structures and slides ahead on the lateral crus of the alar cartilage, forcing it into a caudal rotation. The concavity of the plica nasi is replaced by a thick vertical nostril web, armed by the caudal border of the crus lateral and the bulky myrtiform muscle that Victor Veau called the "muscle of the cleft," which, he advised surgeons, should be sectioned to allow for advancement of the lateral borders of the cleft toward the median line. Having done this, as they reinsert the lateral nasal muscles, surgeons need to guard against attaching the myrtiform muscle on the median line, because, as the support of the nostril web, it would pull it like a curtain toward the nasal septum, blocking the nasal orifice and raising the nasal base. Surgeons must guard against falling into this trap, which by itself could prevent the nursing infant from effectuating nasal breathing. 


\section{3 - 2 - The dynamics of fetal ventilation $^{20}$}

With studies of the Doppler effect showing liquid flux in utero researchers have become acutely aware of the dynamics of fetal ventilation. In collaboration with his wife Claude, Jacques Talmant used ultrasound as a principal tool for studying fetal nasal ventilation, which begins at the $11^{\text {th }}$ week of intrauterine life, perhaps even earlier at the $9^{\text {th }}$ or $10^{\text {th }}$ week. This activity is not as permanent as ventilation in open air because fetal hematosis takes place in the placenta. Nevertheless, infants at the time of birth have already been breathing in utero for six months at a sustained rate of 50 movements per minute. Amniotic fluid in which they accomplish this is incompressible and 800 times denser than air, which conveys to fetal ventilation a forcible influence on the morphogenesis of the respiratory tree from the nose to the lungs. At each ventilatory ejection against the nasal vault the amniotic fluid propels the nasal capsule and in the same movement carries with it the maxilla, to which it is tied by Latham's septo-premaxillary ligament. The braking zone of the amniotic fluid extends by each impact and in doing so models the convexity of the alar domes and the upper lateral cartilages. At inspiration the flux, shown in the blue Doppler color, shows that the nose acts like the convergingdiverging nozzle with an adjustable collar that Laval first described ${ }^{2}$. In this biological context the nasal valve serves as the adjustable collar.

In utero the forcible sagittal growth of the maxilla occurs, without any doubt, in response to this fetal ventilatory dynamic. The nasal capsule plays only a passive intermediate role in transforming the propulsion of the amniotic fluid into a traction force acting on the maxilla. Animated by an alternating movement, the septum grooves and models the vomero-premaxilla splint but it has not originated this movement. Its growth merely accompanies the actual originating force, dynamic ventilation. Thus Scott's theory ${ }^{16}$ becomes subject to very serious question and Moss's functional matrix hypothesis ${ }^{10}$ is forcibly supported and reinforced.

\section{$3-3$ - The influence of the cleft on fetal ventilation ${ }^{27}$}

The loss of pressure from amniotic fluid in the cleft nasal fossa is clearly the cause of the weakness of the projection on this side and, accordingly its hypoplasia. The collapse of the upper lateral cartilage, the alar dome, and all structures on the side of the cleft can be explained in the same way. The gradient of pressure between the two nasal fossae has other consequences. The septum and the vomer are pressed quite strongly throughout their lengths toward the divided nasal fossae, with a septo-vomerian luxation along the length of the splint, and on the opposing side the excess pressure inflates the contralateral nasal fossa where the strong amniotic current raises the whole length of the lateral wall of excessive folds, which, pushed toward the median line become the inferior and middle hypertrophic turbinates occupying the entire nasal fossa. This type of deformation occurs only with unilateral clefts.

There is no longer any doubt about the essentially secondary character of 
the malformation in unilateral clefts and this, more than ever, puts into context the supposed mesodermal deficit as being the source of the cleft, of its hypoplasia, and its "limited and unpredictable" growth potential. Mooney ${ }^{9}$ had shown, much earlier, that the mesodermal deficit theory could not explain why stunted development of the premaxilla in fetuses with unilateral clefts did not become detectable until the $14^{\text {th }}$ week of intrauterine life, that is to say 6 to 7 weeks after the appearance of the cleft. It could not, therefore, be essentially primary but secondary instead, and explained chronologically by disturbances in fetal ventilation caused by the cleft, in other words, by growth mechanism operating in abnormal conditions.

\section{4 - NASAL VENTILATION AFTER BIRTH ${ }^{22}$}

In an instant, at birth, the newborn's nasal ventilation switches to an open-air style and the site of hematosis, the transformation of venous to arterial blood, changes from the placenta to the lungs. While the circulation of air for normal people can be nasal, by mouth, or mixed in accordance with the needs of the moment, the morphology, the functional style, and age of the person, the newborn, because of the shortness of its neck does not have an effective oropharynx and has to rely exclusively on its nose for ventilation. It is only after sufficient vertical growth of the neck has taken place that mouth breathing becomes comfortable.

\section{3 - 4 - Bilateral labio-nasal malformation ${ }^{25,26}$}

In bilateral clefts, which are a veritable duplication of unilateral clefts, the projection of the premaxilla magnifies the separation of the alar cartilages on the median line. They remain flattened and move apart from each other in a way similar to the malformations of the most severe unilateral clefts. Harold $\mathrm{McComb}^{8}$ compares the columella to a fully opened zipper. Spread into the tegument of a collapsed and enlarged tip of the nose, the columella can be removed from this position by bringing the alar cartilages together on the median line, a procedure we have been doing for almost 14 years, starting at the same time Cutting ${ }^{3}$ did, which was just after Mulliken ${ }^{11}$ and $\mathrm{McComb}^{8}$ first performed this operation.

It is essential to understand that "optimal ventilation at rest"21, especially during sleep, is nasal. The steady renewal of air sent to the lungs is not the sole function of nasal breathing, which also participates in conditioning pulmonary air by humidifying and reheating it. But, above all, nasal muco$\mathrm{sa}$, as it warms passing air currents, is also cooling cerebral blood in the very important exchanges that take place in the sphenoidal sinus. The most highly performing cerebral thermo-regulation is quite dependent, therefore, on nasal ventilation and it exerts, along other influences, a considerable energetic effect on the nature of sleep which is very energy producing. Thus, nasal 
ventilation influences the quality of sleep, ensuring that it is not depleted by snoring apnea, nightmares, sweating, or sudden and thirsty awakenings, but is calm and reparative, as it "knits up," in Shakespeare's words, "the ravell'd sleave of care," to prepare the sleeper's capacities for the diurnal concentration so necessary for good performance in school.

Nasal ventilation is accompanied by a closed lip posture, a distal position of the tongue that stimulates growth of the palate and maxilla, and adult type swallowing, all of which are key elements necessary for good facial growth.

In 1996 Donald Warren ${ }^{30}$ demonstrated that $75 \%$ of subjects afflicted with total unilateral or bilateral clefts were permanently, or preponderantly, mouth breathers. The consequences of mouth breathing, low tongue posture, persistence of infantile swallowing, craniorachidian, and facial envelope postural changes combine with scar tissue contractions of the lip and palate as well as hypoplasia of the osseous borders of the cleft directly associated with congenital absence of teeth to lead, frequently, to a Class III tendency and excess anterior vertical dimension. The orthodontic closure of a space left by congenitally absent upper incisors, a phenomenon that occurs in 55\% of cleft palate cases, causes a loss of function for the canine on the side of the cleft and a reduction of the rise of the anterior sector of the maxillary arch ${ }^{27}$.

We know that average, or so-called "normal" children, with completely open upper airways free of any obstacle can, simply because of habits like finger sucking, become habitual mouth breathers, an activity that can lead them into a vicious circle that will accumulate hypertrophied adenoidal, tonsillar, and even nasal mucosal thickening, a cycle that only dental orthopedic and myo-facial therapy can break.

\section{$4-1$ - Special aspects of lip and palatal clefts}

In addition to falling victim to the functional and pathological maladies that afflict other children of their age, children treated for lip and palatal clefts $^{27,28}$ have two characteristics that are specific to their condition, the cleft itself and a great likelihood of having post-operative nasal obstacles. This point is capital. In fact, in cases of complete clefts, newborns breathe through the clefts and cannot have any mental representation of their noses. They cannot begin nasal breathing at birth. If the first operation, which establishes a separation between the nose and the oral cavity, creates a nasal obstruction, the child will install a pattern of mouth breathing that is completely unnatural, because newborns naturally and normally breathe through the nose.

This initial programmation of obligatory mouth breathing leaves a cerebral imprint that in our thirty years of experience $^{23}$ we have learned is extremely difficult to eradicate. The best course of action is to prevent its development and to preserve the alternate mode.

Just as language acquisition needs to be encouraged early so it can proceed on a normal path, the 
functions of nasal breathing should be nourished from the time of the first surgical operation in order to prevent the installation of malfunctions and harmful postural adaptions that are so difficult to correct later no matter how successful the secondary surgical procedure may be. Cleft palate chil- dren will not utilize restored nasal permeability if their tongue posture remains low and if they persist in infantile swallowing and if there is a progressive vertical malpositioning of the geniosymphysis when their lips remain parted at rest.

\section{5 - FORMING A NASAL VENTILATION PROTOCOL}

A comprehensive nasal ventilation protocol should include the most appropriate surgical, orthopedic, and orthodontic techniques designed to restore the full range of functions for cleft palate patients, without compromise, and to maintain them from the time of the first surgical procedure. Such a protocol must organize a chronology of procedures in such a way that they will not aggravate existing deformities of structures that are especially fragile in very young patients. It must take advantage of the strong maxillary growth spurt that functions until patients are about two and a half years old and it must control scar formation by abandoning procedures that rely on secondary epithelial formation in graft areas, of flaps in one layer, or denuded osseous surfaces following closure of hard palate defects.

Realizing that growth potential in these patients is in all likelihood normal and that the maxillary deformity affects the borders of the cleft anterior to the premolars where frequently a lateral incisor and some osseous capital are missing, it is essential for the treatment team to keep the incisor-canine sector of the arch symmetrical and in harmony with its mandibular antagonist, which is, initially, normal and can serve as a reference landmark. The treatment team should think beyond the two dimensional view of sagittal facial development provided by a profile cephalogram and envision the patient in three dimensional, even four dimensional terms.

\section{5 - 1 - The transverse dimension}

An intact transverse dimension is an essential component in the maintenance of normal relations between the maxillary and mandibular arches, which in practice means that the treating orthodontist must preserve the space needed for a replacement for a congenitally missing upper lateral incisor in order to maintain the symmetry and the rise of the incisor-canine sector. Keeping in mind the role that dental buds and, later, teeth play in sustaining the width of the piriform orifice and the anterior portion of the nasal floor and at the same time the need for comfortable, unobstructed nasal ventilation, the treatment team must strive to rehabilitate the gingivoalveolar sector split by the cleft.

Posteriorly, the width of the nasal fossae coincides precisely with the 
width of the palatal vault. This means that surgeons must refrain from undertaking surgical procedures to close osseous defects that will allow spontaneous scarring of surfaces denuded laterally along the length of the alveolar arch to permit translation toward the median line of the lateral fibromucosa, or permit secondary formation of epithelium over a free periosteal graft $^{18}$, or a vomer flap in one layer. All these procedures lead to even more severe scarring that it would remain a contracting force throughout the patient's life, irretrievably shrinking the palatal vault no matter what later treatments might be undertaken. But many centers still practice this type of surgery in the belief that pushing back the soft palate is required for the patient's acquisition of good speech skills even if this procedure impairs maxillary growth. They accept this compromise as the lesser of two evils but we believe we have developed techniques that respect all functions and harm none.

Treatment teams dealing with severe burn patients have abandoned all techniques that allow spontaneous scarring because of its terrible sequellae. In view of this stand, how can any caregivers today rely on scar forming techniques in a zone like the palate that must support so many vital functions? It is a region where normal growth is indispensable for proper mastication and good dental occlusion, and, as we now know, for the maintenance of nasal ventilation.

\section{5 - 2 - The time dimension}

Over the last 30 years we have been closing palatal clefts in two planes, using only palatal tissues, without denuding any surfaces, and without leaving any residual fistulas. Our long term successful outcomes confirm the reliability of this procedure, which can be performed on this schedule: the soft palate on 6 monthold patients and the hard palate for 18 month-old patients. It is in this schedule that the fourth dimension of time plays its part, for it accords with the development of facial structures at the point in time and with enough time needed to achieve the best results.

For these patients, traversing infancy with nasal and facial deformities that worsen with the passage of time is an ordeal that cannot be conjured away with a few formulas and some psychological support. To assure for these patients harmonious growth and a seamless integration into all phases of school and social life from the age of 4 on is the major objective of our protocol, the fruit of the slow maturation of our team effort over a 37 year period.

\section{5 - 3 - Describing the protocol}

The protocol that we have been using for the past 14 years for both bilateral and unilateral clefts is being published this year in the Encyclopédie Médico-Chirurgicale (Elsevier-Masson SAS, Paris), Techniques chirurgicales Chirurgie Plastique reconstructrice et esthétique, Talmant Jean Claude, Talmant Jean Christian, Lumineau Jean Pierre. Fentes labiales et palatines. Traitement primaire. reference 45580.2011.

Because of space constraints, we can describe it here only in a highly abbreviated form. 


\section{5-3-1-Preoperative orthopedic treatment}

We have never used passive preoperative orthopedic treatment because in the 60 years that others have employed it no evidence that it confers a durable advantage has ever been demonstrated.

And active early orthopedic treatment is actually harmful! Closing the cleft too soon amounts to removing the possibility of adjusting maxillary width to harmonize with that of the mandible when the temporary dentition is in place thus assuring good canine function.

\section{5-3-2-Patient's age at the time of the first operation}

Many French treatment teams advocate early operations. What should we make of this?

The claim that an early operation has a favorable psychological effect on parents is highly debatable not only for ethical but also for a number of other reasons. The primary justification for the operation is to provide the child with the best possible result not to satisfy maternal impatience as a high priority. Operations performed too early deform maxillas that are only slightly calcified in 6 month-old children. Ross ${ }^{14}$ showed that growth defects occur more frequently in children who were operated on initially before they were 4 months old. Surgeons in Great Britain have stopped performing early operations because they were getting poor results with them. The supposed advantage that this precocious procedure produces "good" scarring of the fetal type has never been proven. And the most recent studies of the use of general anesthesia for neonates $1,5,6$ suggest that operations should not be undertaken on children in the first three months of post-natal life except in cases of urgent emergencies. Another convincing argument is that the most functional type of surgery is quite complex and cannot successfully be performed on children under the age of 3 months.

\section{5 - 3 - 3 - Closing lip, nasal, and soft palate clefts on 6 month-old children}

\section{- Closing the soft palate cleft}

We begin the first operation by closing the soft palate cleft using the Sommerlad technique ${ }^{17}$. We dissect the elevator muscles of the soft palate almost completely and transpose them to reconstruct a posterior muscular ring free of any abnormal attachment on the osseous borders of the palatal cleft. Following this procedure the soft palate can perform far more effectively.

\section{- Closing the labio-nasal cleft}

We repair the lip using Millard's method, which leaves a vertical scar on the philtral crest. We make lateral mobilization possible with an extended sub-periosteal dissection on the anterior surface of the maxilla. While suturing muscles on three planes we pay particular attention to the deep plane of the myrtiform muscle, which should not be reinserted on the anterior nasal spine to prevent the vertical nostril web from completely obstructing the nasal valve. 


\section{- Nasal correction}

We finish correcting the nostrils when we close the cleft lip by liberating the sub-perichondrial tissue of the nasal septum and the upper lateral cartilage and with a sub-cutaneous dissection of the alar cartilage.

We make the final repositioning with a nasal conformer modeled during the procedure, which is replaced 6 days later by a removable conformer that is worn for four months. This precaution radically improves the functional and esthetic quality of the results. All the young patients we see on a 4 month check-up visit are sleeping, their parents report, with their lips closed.

\section{$5-3-4$ - Closing the residual osseous palatal cleft at 18 months}

During the year following the first operation the changes in the residual osseous palatal cleft are surprising, with a spontaneous reduction in its size of such proportions that surgeons can always close it when patients are 18 months old, in two planes, simply by sliding the parts, without leaving a denuded osseous zone throughout the length of the alveolar arch. The outcome is simple and leaves a palatal vault free of contractile scar tissue.

\section{5 - 3 - 5 - Closing the gingivo- alveolar cleft when patients are 4 to 5 years-old}

We close gingivo-alveolar clefts when patients are 4 to 5 years old. We can evaluate the occlusion and, especially, canine function when children are about 3 and a half years old.
Orthopedic preparation is particularly effective at that age when it is possible to expand the maxillary maxilla with a quadhelix $\left(8\right.$ by $\left.10^{\mathrm{e}}\right)$ affixed to the temporary molars with Rocky Mountain (4 by $10^{\mathrm{e}}$ ) peri-dental steel ligature wire. We undertake this expansion for all patients whose upper intercanine is not at least $4 \mathrm{~mm}$ greater than the mandibular intercanine distance, which is always normal at that age. Because the appliance that accomplishes this expansion is firmly ligated it has a direct effect on basal bone, thus re-establishing normal width for the piriform orifice and the nasal fossae.

We then close the cleft by gingivoperiosteoplasty and a bone graft. In this way we have rehabilitated the gingivo-alveolar arch leaving it in an ideal periodontal condition for the eruption of teeth that can be mobilized for orthodontic treatment and without any residual gingival fistulas.

\section{5-3-6- Follow-up of primary treatment}

We leave the quadhelix in place until the six year molars erupt then, if necessary, replace it with another quadhelix anchored by bands with its midline aligned with erupting teeth. This procedure works out as classic orthodontic therapy adjusted to compensate for the particularity of frequent agenesis of upper lateral incisors whose space must be zealously maintained so that growth can proceed normally with sufficient space preserved for the piriform orifice. This mechano-therapy must also produce a symmetrical arch form and good contour of the incisive area that will lead to a stable occlusion. 


\section{6 - CONCLUSION}

From the vantage point of 14 years after its implementation, we believe we can reasonably hope to demonstrate by study of consecutive cases that, by using our protocol that concentrates on ameliorating nasal ventilation from the earliest possible age, patients developed facial growth rates very close to normal and required no special appliances other than implants to replace congenitally absent upper lateral incisors. Indications of need for orthognathic surgery have been quite rare for our cadre of patients, far less than have been recorded for other patients suffering from similar labiomaxillary-palatal clefts where rates of Lefort Lefort 1 osteotomies of $25 \%$ for unilateral clefts and 35 to $45 \%$ for bilateral clefts have been reported as routine by excellent treatment teams.

The benefits of nasal ventilation for the great majority of these children are already evident, as shown by the quality of their development in every area, phonation, occlusion, growth, and appearance.

\section{REFERENCES}

1. Brambrink AM. et al. Isoflurane-induced neuroapoptosis in the neonatal Rhesus Macaque Brain. Anesthesiology 2010;112:834-41.

2. Comolet R. Ecoulement dans les singularités, Pertes de charges singulières. In :

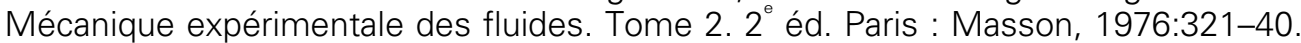

3. Cutting C, Grayson B, Brecht L, Santiago P, Wood R, Kwon S. Presurgical columellar elongation and primary retrograde nasal reconstruction in one-stage bilateral cleft lip and nose repair. Plast Reconstr Surg 1998;101:630-9.

4. Delaire J. La chéilo-rhinoplastie primaire pour fentes labio-maxillaires congénitales unilatérales. Essai de schématisation d'une technique. Rev Stomatol Chir Maxillofac 1975;76:193-215.

5. Editorial Views. Anesthesia and neurodevelopment in children: time for an answer? Anesthesiology 2008;109:757-61.

6. Editorial Views. Anesthetic effect on the developing brain: insights from epidemiology. Anesthesiology 2009;110:1-3.

7. Diah E, Lo LJ, Huang CS, Sudjatmiko G, Susanto I, Chen YR. Maxillary growth of adult patients with unoperated cleft: answers to the debates. J Plast Reconstr Aesthet Surg 2007;60:407-13.

8. McComb H. Primary repair of the bilateral cleft lip nose: a 4 year review. Plast Reconstr Surg 1994; 94:37-47.

9. Mooney MP, Siegel MI, Kimes KR, Todhunter J. Premaxillary development in normal and cleft lip and palate human fetuses using three-dimensional computer reconstruction. Cleft Palate Craniofac J 1991;28:49-54.

10. Moss ML, Bromberg BE, Song IG, Eisenman G. The passive role of nasal septal cartilage in mid-facial growth. Plast Reconstr Surg 1968;41:536-42.

11. Mulliken JB. Correction of the bilateral cleft lip nasal deformity: evolution of a surgical concept. Cleft Palate Craniofacial J 1992;2540-5.

12. Ortiz-Monasterio F, Rebeil AS, Valderama M, Cruz R. Cephalometric measurements on adult patients with non-operated cleft palates. Plast Reconstr Surg Transplant Bull 1959;24;53-61. 
13. Raphaël B, Morand B, Bettega G, Lesne C, Lesne V. Evaluation à long terme de I'uranoplastie par greffe de périoste tibial dans la fente labio-maxillo-palatine totale unilatérale. A propos de 51 cas cliniques. Ann Chir Plast Esthet 2002;47;196-203.

14. Ross RB. Treatment variables affecting facial growth in complete unilateral cleft lip and palate. Cleft Palate J 1987;24:5-77.

15. Rullo R, Laino G, Cataneo M, Mazzarella N, Festa VM, Gombos F. Conséquences de la chéilorhinoplastie de Delaire sur la croissance de la face moyenne chez les patients avec fente labiale et palatine unilatérale. Eur J Orthod 2009;31:64-7.

16. Scott JH. The cartilage of the nasal septum. Brit Dent J 1953;95:37-44.

17. Sommerlad BC. A technique for cleft palate repair. Plast Reconstr Surg 2003;112:1542-8.

18. Stricker M, Chancholle AR, Flot F, Malka G, Montoya A. La greffe périostée dans la réparation de la fente totale du palais primaire. Ann Chir Plast 1977;22:117-25.

19. Talmant J, Talmant JC, Deniaud J. Mécanique de I'enveloppe faciale. 3 Organisation structurale de l'enveloppe. Rev Orthop Dento Faciale 2000;34;213-43.

20. Talmant J, Deniaud J, Nivet MH. Ventilation fœtale, ventilation postnatale et morphogénèse. In : La dimension verticale. 1. Ventilation nasale et dimension verticale : bases morphologiques et physiologiques. Orthod Fr 2003;74(2):147-200.

21. Talmant J, Deniaud J, Nivet MH. Définition de la "ventilation nasale optimale». In : La dimension verticale. 1. Ventilation nasale et dimension verticale : bases morphologiques et physiologiques. Orthod Fr 2003;74(2):201-25.

22. Talmant J, Deniaud J. Approche actuelle du traitement des troubles de la ventilation nasale de l'enfant et de l'adolescent. Rev Orthop Dento Faciale 2010;44:285-302.

23. Talmant JC. La narine du bec-de-lièvre unilatéral. Peut-on concilier esthétique et ventilation normale ? Orthod Fr 1982;53:451-6.

24. Talmant JC. Nasal malformation associated with unilateral cleft lip. Scand J Plast Reconstr Surg Hand Surg 1993;27;183-91.

25. Talmant JC. Cleft rhinoplasty. In: Ward Booth P, Schendel SA, Hausamen JE, ed. Maxillofacial surgery. $1^{\text {st }}$ edition. London: Churchill Livingstone, 1999:1133-71.

26. Talmant JC. Current trends in the treatment of bilateral cleft lip and palate. In: Precious David S, Cleft Lip and Palate: A physiological approach. Oral Maxillofac Surg Clin North Am 2000;12;421-41.

27. Talmant J.C. Evolution of the functional repair concept for cleft lip and palate patients. Indian J Plast Surg 2006,39:196-209.

28. Talmant JC, Talmant JC, Lumineau JP. Une approche fonctionnelle lors du traitement primaire des fentes labioalvéolopalatovélaires pour le minimum de séquelles. Rev Stomatol Chir Maxillofac 2007;108:255-63.

29. Veau V. Étude anatomique du bec-de-lièvre unilatéral total. Annales d'Anatomie Pathologique et d'Anatomie Normale 1928;5:601-32.

30. Warren DW, Drake AF, Davis JU. The nasal airway in breathing and speech. In: Berkowitz S. ed. Cleft lip and palate, perspectives in management. San Diego: Singular Publishing Group Inc, 1996; vol Il:61-73. 